\title{
Stability Analysis of a System of Exponential Difference Equations
}

\author{
Q. Din, ${ }^{1}$ K. A. Khan, ${ }^{2}$ and A. Nosheen ${ }^{2}$ \\ ${ }^{1}$ Department of Mathematics, University of Poonch Rawalakot, Rawalakot 12350, Pakistan \\ ${ }^{2}$ Department of Mathematics, University of Sargodha, Sargodha 40100, Pakistan
}

Correspondence should be addressed to Q. Din; qamar.sms@gmail.com

Received 14 May 2014; Accepted 14 July 2014; Published 24 July 2014

Academic Editor: Garyfalos Papashinopoulos

Copyright (C) 2014 Q. Din et al. This is an open access article distributed under the Creative Commons Attribution License, which permits unrestricted use, distribution, and reproduction in any medium, provided the original work is properly cited.

\begin{abstract}
We study the boundedness character and persistence, existence and uniqueness of positive equilibrium, local and global behavior, and rate of convergence of positive solutions of the following system of exponential difference equations: $x_{n+1}=\left(\alpha_{1}+\beta_{1} e^{-x_{n}}+\right.$ $\left.\gamma_{1} e^{-x_{n-1}}\right) /\left(a_{1}+b_{1} y_{n}+c_{1} y_{n-1}\right), y_{n+1}=\left(\alpha_{2}+\beta_{2} e^{-y_{n}}+\gamma_{2} e^{-y_{n-1}}\right) /\left(a_{2}+b_{2} x_{n}+c_{2} x_{n-1}\right)$, where the parameters $\alpha_{i}, \beta_{i}, \gamma_{i}, a_{i}, b_{i}$, and $c_{i}$ for $i \in\{1,2\}$ and initial conditions $x_{0}, x_{-1}, y_{0}$, and $y_{-1}$ are positive real numbers. Furthermore, by constructing a discrete Lyapunov function, we obtain the global asymptotic stability of the positive equilibrium. Some numerical examples are given to verify our theoretical results.
\end{abstract}

\section{Introduction}

Many population models are governed by exponential difference equations. We refer to [1-6] and the references therein. Systems of nonlinear difference equations of higher-order are of paramount importance in applications. Such equations also appear naturally as discrete analogues and as numerical solutions of systems differential and delay differential equations which model diverse phenomena in biology, ecology, physiology, physics, engineering, and economics. For applications and basic theory of rational difference equations we refer to [7-9]. In [10-17] applications of difference equations in mathematical biology are given. It is very interesting to investigate the behavior of solutions of a system of nonlinear difference equations and to discuss the local asymptotic stability of their equilibrium points.

El-Metwally et al. [1] investigated boundedness character, asymptotic behavior, periodicity nature of the positive solutions, and stability of equilibrium point of the following population model:

$$
x_{n+1}=\alpha+\beta x_{n-1} e^{-x_{n}} .
$$

Papaschinopoulos et al. [2] studied the boundedness, the persistence, and the asymptotic behavior of positive solutions of the following two directional interactive and invasive species models:

$$
x_{n+1}=a+b x_{n-1} e^{-y_{n}}, \quad y_{n+1}=c+d y_{n-1} e^{-x_{n}} .
$$

Papaschinopoulos et al. [3] investigated the asymptotic behavior of the solutions of the following three systems of difference equations of exponential form:

$$
\begin{array}{ll}
x_{n+1}=\frac{\alpha+\beta e^{-y_{n}}}{\gamma+y_{n-1}}, & y_{n+1}=\frac{\delta+\epsilon e^{-x_{n}}}{\zeta+x_{n-1}}, \\
x_{n+1}=\frac{\alpha+\beta e^{-y_{n}}}{\gamma+x_{n-1}}, & y_{n+1}=\frac{\delta+\epsilon e^{-x_{n}}}{\zeta+y_{n-1}}, \\
x_{n+1}=\frac{\alpha+\beta e^{-x_{n}}}{\gamma+y_{n-1}}, & y_{n+1}=\frac{\delta+\epsilon e^{-y_{n}}}{\zeta+x_{n-1}} .
\end{array}
$$

Recently, Papaschinopoulos and Schinas [4] studied the asymptotic behavior of the positive solutions of the systems of the two difference equations:

$$
\begin{array}{ll}
x_{n+1}=a+b y_{n-1} e^{-y_{n}}, & y_{n+1}=c+d x_{n-1} e^{-x_{n}}, \\
x_{n+1}=a+b y_{n-1} e^{-x_{n}}, & y_{n+1}=c+d x_{n-1} e^{-y_{n}} .
\end{array}
$$


Motivated by the above study, our aim in this paper is to investigate the qualitative behavior of positive solutions of the following system of exponential difference equations:

$$
\begin{aligned}
& x_{n+1}=\frac{\alpha_{1}+\beta_{1} e^{-x_{n}}+\gamma_{1} e^{-x_{n-1}}}{a_{1}+b_{1} y_{n}+c_{1} y_{n-1}}, \\
& y_{n+1}=\frac{\alpha_{2}+\beta_{2} e^{-y_{n}}+\gamma_{2} e^{-y_{n-1}}}{a_{2}+b_{2} x_{n}+c_{2} x_{n-1}},
\end{aligned}
$$

where the parameters $\alpha_{i}, \beta_{i}, \gamma_{i}, a_{i}, b_{i}$, and $c_{i}$ for $i \in\{1,2\}$ and initial conditions $x_{0}, x_{-1}, y_{0}$, and $y_{-1}$ are positive real numbers.

More precisely, we investigate the boundedness character and persistence, existence and uniqueness of positive steady state, local asymptotic stability and global behavior of unique positive equilibrium point, and rate of convergence of positive solutions of system (5) which converge to its unique positive equilibrium point. Some special cases of system (5) can be treated as population models of two species [3].

\section{Main Results}

The following theorem shows that every solution of (5) is bounded and persists.

Theorem 1. Every positive solution $\left\{\left(x_{n}, y_{n}\right)\right\}$ of system (5) is bounded and persists.

Proof. For any positive solution $\left\{\left(x_{n}, y_{n}\right)\right\}$ of system (5), one has

$$
\begin{array}{r}
x_{n+1} \leq \frac{\alpha_{1}+\beta_{1}+\gamma_{1}}{a_{1}}=U_{1}, \\
y_{n+1} \leq \frac{\alpha_{2}+\beta_{2}+\gamma_{2}}{a_{2}}=U_{2}, \\
n=0,1,2, \ldots
\end{array}
$$

Furthermore, from systems (5) and (6) we obtain that

$$
\begin{array}{r}
x_{n+1} \geq \frac{\alpha_{1}+\beta_{1} e^{-U_{1}}+\gamma_{1} e^{-U_{1}}}{a_{1}+b_{1} U_{2}+c_{1} U_{2}}=L_{1}, \\
y_{n+1} \geq \frac{\alpha_{2}+\beta_{2} e^{-U_{2}}+\gamma_{2} e^{-U_{2}}}{a_{2}+b_{2} U_{1}+c_{2} U_{1}}=L_{2}, \\
n=2,3, \ldots
\end{array}
$$

From (6) and (7), it follows that

$$
L_{1} \leq x_{n} \leq U_{1}, \quad L_{2} \leq y_{n} \leq U_{2}, \quad n=3,4, \ldots
$$

Hence, the theorem is proved.

Lemma 2. Let $\left\{\left(x_{n}, y_{n}\right)\right\}$ be a positive solution of system (5). Then, $\left[L_{1}, U_{1}\right] \times\left[L_{2}, U_{2}\right]$ is invariant set for system (5).

Proof. The proof follows by induction.
2.1. Stability Analysis. Let us consider four-dimensional discrete dynamical system of the form:

$$
\begin{aligned}
& x_{n+1}=f\left(x_{n}, x_{n-1}, y_{n}, y_{n-1}\right) \\
& y_{n+1}=g\left(x_{n}, x_{n-1}, y_{n}, y_{n-1}\right), \quad n=0,1, \ldots,
\end{aligned}
$$

where $f: I^{2} \times J^{2} \rightarrow I$ and $g: I^{2} \times J^{2} \rightarrow J$ are continuously differentiable functions and $I, J$ are some intervals of real numbers. Furthermore, a solution $\left\{\left(x_{n}, y_{n}\right)\right\}_{n=-1}^{\infty}$ of system (9) is uniquely determined by initial conditions $\left(x_{i}, y_{i}\right) \in$ $I \times J$ for $i \in\{-1,0\}$. Along with system (9) we consider the corresponding vector map $F=\left(f, x_{n}, g, y_{n}\right)$. An equilibrium point of (9) is a point $(\bar{x}, \bar{y})$ that satisfies

$$
\begin{aligned}
& \bar{x}=f(\bar{x}, \bar{x}, \bar{y}, \bar{y}), \\
& \bar{y}=g(\bar{x}, \bar{x}, \bar{y}, \bar{y}) .
\end{aligned}
$$

The point $(\bar{x}, \bar{y})$ is also called a fixed point of the vector map F.

Definition 3. Let $(\bar{x}, \bar{y})$ be an equilibrium point of the system (9).

(i) An equilibrium point $(\bar{x}, \bar{y})$ is said to be stable if for every $\varepsilon>0$ there exists $\delta>0$ such that for every initial condition $\left(x_{i}, y_{i}\right), i \in\{-1,0\}$, if $\| \sum_{i=-1}^{0}\left(x_{i}, y_{i}\right)-$ $(\bar{x}, \bar{y}) \|<\delta$ implies $\left\|\left(x_{n}, y_{n}\right)-(\bar{x}, \bar{y})\right\|<\varepsilon$ for all $n>0$, where $\|\cdot\|$ is usual Euclidian norm in $\mathbb{R}^{2}$.

(ii) An equilibrium point $(\bar{x}, \bar{y})$ is said to be unstable if it is not stable.

(iii) An equilibrium point $(\bar{x}, \bar{y})$ is said to be asymptotically stable if there exists $\eta>0$ such that $\left\|\sum_{i=-1}^{0}\left(x_{i}, y_{i}\right)-(\bar{x}, \bar{y})\right\|<\eta$ and $\left(x_{n}, y_{n}\right) \rightarrow(\bar{x}, \bar{y})$ as $n \rightarrow \infty$.

(iv) An equilibrium point $(\bar{x}, \bar{y})$ is called global attractor if $\left(x_{n}, y_{n}\right) \rightarrow(\bar{x}, \bar{y})$ as $n \rightarrow \infty$.

(v) An equilibrium point $(\bar{x}, \bar{y})$ is called asymptotic global attractor if it is a global attractor and stable.

Definition 4. Let $(\bar{x}, \bar{y})$ be an equilibrium point of a map $F=$ $\left(f, x_{n}, g, y_{n}\right)$, where $f$ and $g$ are continuously differentiable functions at $(\bar{x}, \bar{y})$. The linearized system of (9) about the equilibrium point $(\bar{x}, \bar{y})$ is

$$
X_{n+1}=F\left(X_{n}\right)=F_{J} X_{n},
$$

where $X_{n}=\left(\begin{array}{c}x_{n} \\ y_{n} \\ x_{n-1} \\ y_{n-1}\end{array}\right)$ and $F_{J}$ is Jacobian matrix of system about the equilibrium point $(\bar{x}, \bar{y})$.

To construct corresponding linearized form of system (5) we consider the following transformation:

$$
\left(x_{n}, y_{n}, x_{n-1}, y_{n-1}\right) \longmapsto\left(f, g, f_{1}, g_{1}\right),
$$

where $f=x_{n+1}, g=y_{n+1}, f_{1}=x_{n}$, and $g_{1}=y_{n}$. The linearized system of (5) about $(\bar{x}, \bar{y})$ is given by

$$
Z_{n+1}=F_{J}(\bar{x}, \bar{y}) Z_{n}
$$


where $Z_{n}=\left(\begin{array}{c}x_{n} \\ y_{n} \\ x_{n-1} \\ y_{n-1}\end{array}\right)$ and the Jacobian matrix about the fixed point $(\bar{x}, \bar{y})$ under transformation (12) is given by

$$
F_{J}(\bar{x}, \bar{y})=\left(\begin{array}{cccc}
A_{1} & A_{2} & A_{3} & A_{4} \\
B_{1} & B_{2} & B_{3} & B_{4} \\
1 & 0 & 0 & 0 \\
0 & 1 & 0 & 0
\end{array}\right)
$$

where

$$
\begin{aligned}
& A_{1}=-\frac{\beta_{1} e^{-\bar{x}}}{a_{1}+\left(b_{1}+c_{1}\right) \bar{y}}, \\
& A_{2}=-\frac{b_{1}\left(\alpha_{1}+\left(\beta_{1}+\gamma_{1}\right) e^{-\bar{x}}\right)}{\left(a_{1}+\left(b_{1}+c_{1}\right) \bar{y}\right)^{2}}, \\
& A_{3}=-\frac{\gamma_{1} e^{-\bar{x}}}{a_{1}+\left(b_{1}+c_{1}\right) \bar{y}}, \\
& A_{4}=-\frac{c_{1}\left(\alpha_{1}+\left(\beta_{1}+\gamma_{1}\right) e^{-\bar{x}}\right)}{\left(a_{1}+\left(b_{1}+c_{1}\right) \bar{y}\right)^{2}} \\
& B_{1}=-\frac{b_{2}\left(\alpha_{2}+\left(\beta_{2}+\gamma_{2}\right) e^{-\bar{y}}\right)}{\left(a_{2}+\left(b_{2}+c_{2}\right) \bar{x}\right)^{2}} \\
& B_{2}=-\frac{\beta_{2} e^{-\bar{y}}}{a_{2}+\left(b_{2}+c_{2}\right) \bar{x}}, \\
& B_{4}=-\frac{\gamma_{2} e^{-\bar{y}}}{a_{2}+\left(b_{2}+c_{2}\right) \bar{x}} \cdot\left(\alpha_{2}+\left(\beta_{2}+\gamma_{2}\right) e^{-\bar{y}}\right) \\
& B_{3}=-\frac{\left(a_{2}+\left(b_{2}+c_{2}\right) \bar{x}\right)^{2}}{c_{2}}
\end{aligned}
$$

Lemma 5 (see [9]). Assume that $X_{n+1}=F\left(X_{n}\right), n=0,1, \ldots$, is a system of difference equations such that $\bar{X}$ is a fixed point of $F$. If all eigenvalues of the Jacobian matrix $J_{F}$ about $\bar{X}$ lie inside the open unit disk $|\lambda|<1$, then $\bar{X}$ is locally asymptotically stable. If one of them has a modulus greater than one, then $\bar{X}$ is unstable.

Theorem 6. System (5) has a unique positive equilibrium point $(\bar{x}, \bar{y}) \in\left[L_{1}, U_{1}\right] \times\left[L_{2}, U_{2}\right]$, if the following condition is satisfied:

$$
a_{2}+L_{1}\left(b_{2}+c_{2}\right)<\frac{\alpha_{2}+\left(\beta_{2}+\gamma_{2}\right) e^{-K}}{K}
$$

where

$$
K=\frac{\alpha_{1}+e^{-L_{1}}\left(\beta_{1}+\gamma_{1}\right)-a_{1} L_{1}}{L_{1}\left(b_{1}+c_{1}\right)} .
$$

Proof. Consider the following system of equations:

$$
x=\frac{\alpha_{1}+\left(\beta_{1}+\gamma_{1}\right) e^{-x}}{a_{1}+\left(b_{1}+c_{1}\right) y}, \quad y=\frac{\alpha_{2}+\left(\beta_{2}+\gamma_{2}\right) e^{-y}}{a_{2}+\left(b_{2}+c_{2}\right) x} .
$$

From (18), it follows that

$$
\begin{aligned}
& y=\frac{\alpha_{1}+\left(\beta_{1}+\gamma_{1}\right) e^{-x}-a_{1} x}{\left(b_{1}+c_{1}\right) x}, \\
& x=\frac{\alpha_{2}+\left(\beta_{2}+\gamma_{2}\right) e^{-y}-a_{2} y}{\left(b_{2}+c_{2}\right) y} .
\end{aligned}
$$

Set

$$
F(x)=\frac{\alpha_{2}+\left(\beta_{2}+\gamma_{2}\right) e^{-f(x)}-a_{2} f(x)}{\left(b_{2}+c_{2}\right) f(x)}-x,
$$

where $f(x)=\left(\alpha_{1}+\left(\beta_{1}+\gamma_{1}\right) e^{-x}-a_{1} x\right) /\left(\left(b_{1}+c_{1}\right) x\right)$ and $x \in$ $\left[L_{1}, U_{1}\right]$. Then, it follows that

$$
\begin{gathered}
F\left(L_{1}\right)=L_{1}\left(b_{1}+c_{1}\right) \\
\times\left(\alpha_{2}+\left(\beta_{2}+\gamma_{2}\right) e^{-\left(\alpha_{1}+e^{-L_{1}}\left(\beta_{1}+\gamma_{1}\right)-a_{1} L_{1}\right) / L_{1}\left(b_{1}+c_{1}\right)}\right. \\
\left.\quad-\frac{a_{2}\left(\alpha_{1}+e^{-L_{1}}\left(\beta_{1}+\gamma_{1}\right)-a_{1} L_{1}\right)}{L_{1}\left(b_{1}+c_{1}\right)}\right) \\
\times\left(\left(b_{2}+c_{2}\right)\left(\alpha_{1}+e^{-L_{1}}\left(\beta_{1}+\gamma_{1}\right)-a_{1} L_{1}\right)\right)^{-1}-L_{1} .
\end{gathered}
$$

Furthermore, it is easy to see that

$$
\begin{gathered}
\left(a_{1}+\left(b_{1}+c_{1}\right) U_{2}\right)\left(\alpha_{1}+e^{-L_{1}}\left(\beta_{1}+\gamma_{1}\right)\right) \\
>a_{1}\left(\alpha_{1}+\left(\beta_{1}+\gamma_{1}\right) e^{-U_{1}}\right) .
\end{gathered}
$$

From (22) it follows that

$$
\alpha_{1}+e^{-L_{1}}\left(\beta_{1}+\gamma_{1}\right)-a_{1} L_{1}>0 .
$$

Let $K=\left(\alpha_{1}+e^{-L_{1}}\left(\beta_{1}+\gamma_{1}\right)-a_{1} L_{1}\right) /\left(L_{1}\left(b_{1}+c_{1}\right)\right)>0$. Then $F\left(L_{1}\right)$ can be expressed as

$$
F\left(L_{1}\right)=\frac{\alpha_{2}+\left(\beta_{2}+\gamma_{2}\right) e^{-K}-a_{2} K}{\left(b_{2}+c_{2}\right) K}-L_{1} \text {. }
$$

Suppose that $a_{2}+L_{1}\left(b_{2}+c_{2}\right)<\left(\alpha_{2}+\left(\beta_{2}+\gamma_{2}\right) e^{-K}\right) / K$; then, it follows that $F\left(L_{1}\right)>0$. Furthermore, we have

$$
\begin{gathered}
F\left(U_{1}\right)=U_{1}\left(b_{1}+c_{1}\right)\left(\left(\beta_{2}+\gamma_{2}\right) e^{\left(\beta_{1}+\gamma_{1}\right)\left(1-e^{-U_{1}}\right) / U_{1}\left(b_{1}+c_{1}\right)}\right. \\
\left.+\alpha_{2}+\frac{a_{2}\left(\beta_{1}+\gamma_{1}\right)\left(1-e^{-U_{1}}\right)}{U_{1}\left(b_{1}+c_{1}\right)}\right) \\
\quad \times\left(\left(b_{2}+c_{2}\right)\left(\alpha_{1}+e^{-U_{1}}\left(\beta_{1}+\gamma_{1}\right)-a_{1} U_{1}\right)\right)^{-1}-U_{1} .
\end{gathered}
$$

Then it is easy to see that

$$
\alpha_{1}+e^{-U_{1}}\left(\beta_{1}+\gamma_{1}\right)-a_{1} U_{1}=\left(\beta_{1}+\gamma_{1}\right)\left(e^{-U_{1}}-1\right)<0,
$$


which gives that $F\left(U_{1}\right)<0$. Hence, $F(x)=0$ has at least one positive solution in $\left[L_{1}, U_{1}\right]$. Moreover, we obtain that

$$
\begin{aligned}
F^{\prime}(x)= & -1-\frac{f^{\prime}(x)}{\left(b_{2}+c_{2}\right) f(x)} \\
& \times\left[a_{2}+\left(b_{2}+c_{2}\right) x+\left(\beta_{2}+\gamma_{2}\right) e^{-f(x)}\right],
\end{aligned}
$$

where

$$
f^{\prime}(x)=-\frac{\alpha_{1}+\left(\beta_{1}+\gamma_{1}\right)(1+x) e^{-x}}{\left(b_{1}+c_{1}\right) x^{2}} .
$$

Then, from (28) it follows that $f\left(U_{1}\right)<f(x)<f\left(L_{1}\right)$ and using (26) we obtain

$$
\begin{aligned}
F^{\prime}(x)= & -1+\frac{\alpha_{1}+\left(\beta_{1}+\gamma_{1}\right)(1+x) e^{-x}}{\left(b_{1}+c_{1}\right)\left(b_{2}+c_{2}\right) x^{2} f(x)} \\
& \times\left[a_{2}+\left(b_{2}+c_{2}\right) x+\left(\beta_{2}+\gamma_{2}\right) e^{-f(x)}\right] \\
\leq & -1+\frac{\alpha_{1}+\left(\beta_{1}+\gamma_{1}\right)(1+x) e^{-x}}{\left(b_{1}+c_{1}\right)\left(b_{2}+c_{2}\right) x^{2} f\left(U_{1}\right)} \\
& \times\left[a_{2}+\left(b_{2}+c_{2}\right) x+\left(\beta_{2}+\gamma_{2}\right) e^{-f(x)}\right]<0 .
\end{aligned}
$$

Hence, $F(x)=0$ has a unique positive solution in $\left[L_{1}, U_{1}\right]$. The proof is therefore completed.

Theorem 7. The unique positive equilibrium point of system (5) is locally asymptotically stable under the following condition:

$$
\begin{aligned}
& \frac{\beta_{1} e^{-L_{1}}}{a_{1}+\left(b_{1}+c_{1}\right) L_{2}}+\left(1+\frac{\beta_{1} e^{-L_{1}}}{a_{1}+\left(b_{1}+c_{1}\right) L_{2}}\right) \\
& \times\left(\frac{c_{2}\left(\alpha_{2}+\left(\beta_{2}+\gamma_{2}\right) e^{-L_{2}}\right)}{\left(a_{2}+\left(b_{2}+c_{2}\right) L_{1}\right)^{2}}+\frac{\left(\beta_{2}+\gamma_{2}\right) e^{-L_{2}}}{a_{2}+\left(b_{2}+c_{2}\right) L_{1}}\right) \\
& +\frac{b_{2}\left(\alpha_{2}+\left(\beta_{2}+\gamma_{2}\right) e^{-L_{2}}\right)}{\left(a_{2}+\left(b_{2}+c_{2}\right) L_{1}\right)^{2}} \\
& \times\left(\frac{\left(b_{1}+c_{1}\right)\left(\alpha_{1}+\left(\beta_{1}+\gamma_{1}\right) e^{-L_{1}}\right)}{\left(a_{1}+\left(b_{1}+c_{1}\right) L_{2}\right)^{2}}\right. \\
& \left.+\frac{\gamma_{1} e^{-L_{1}}}{a_{1}+\left(b_{1}+c_{1}\right) L_{2}}\right)<1 .
\end{aligned}
$$

Proof. The characteristic polynomial of Jacobian matrix $F_{J}(\bar{x}, \bar{y})$ about $(\bar{x}, \bar{y})$ is given by

$$
\begin{aligned}
P(\lambda)= & \lambda^{4}-\left(A_{1}+B_{2}\right) \lambda^{3}-\left(A_{2} B_{1}-A_{1} B_{2}+B_{3}+B_{4}\right) \lambda^{2} \\
& -\left(\left(A_{3}+A_{4}\right) B_{1}-\left(B_{3}+B_{4}\right) A_{1}\right) \lambda .
\end{aligned}
$$

Clearly, one root of $P(\lambda)$ is 0 . To check the behavior of the other three roots of $P(\lambda)$, we let $\Phi(\lambda)=\lambda^{3}$ and $\Psi(\lambda)=\left(A_{1}+\right.$ $\left.B_{2}\right) \lambda^{2}+\left(A_{2} B_{1}-A_{1} B_{2}+B_{3}+B_{4}\right) \lambda+\left(A_{3}+A_{4}\right) B_{1}-\left(B_{3}+B_{4}\right) A_{1}$. Assume that (30) holds and $|\lambda|=1$; then, one has

$$
\begin{aligned}
& |\Psi(\lambda)|<\left|A_{1}+B_{2}\right|+\left|A_{2} B_{1}-A_{1} B_{2}+B_{3}+B_{4}\right| \\
& +\left|\left(A_{3}+A_{4}\right) B_{1}-\left(B_{3}+B_{4}\right) A_{1}\right| \\
& <\frac{\beta_{1} e^{-\bar{x}}}{a_{1}+\left(b_{1}+c_{1}\right) \bar{y}}+\left(1+\frac{\beta_{1} e^{-\bar{x}}}{a_{1}+\left(b_{1}+c_{1}\right) \bar{y}}\right) \\
& \times\left(\frac{c_{2}\left(\alpha_{2}+\left(\beta_{2}+\gamma_{2}\right) e^{-\bar{y}}\right)}{\left(a_{2}+\left(b_{2}+c_{2}\right) \bar{x}\right)^{2}}+\frac{\left(\beta_{2}+\gamma_{2}\right) e^{-\bar{y}}}{a_{2}+\left(b_{2}+c_{2}\right) \bar{x}}\right) \\
& +\frac{b_{2}\left(\alpha_{2}+\left(\beta_{2}+\gamma_{2}\right) e^{-\bar{y}}\right)}{\left(a_{2}+\left(b_{2}+c_{2}\right) \bar{x}\right)^{2}} \\
& \times\left(\frac{\left(b_{1}+c_{1}\right)\left(\alpha_{1}+\left(\beta_{1}+\gamma_{1}\right) e^{-\bar{x}}\right)}{\left(a_{1}+\left(b_{1}+c_{1}\right) \bar{y}\right)^{2}}\right. \\
& \left.+\frac{\gamma_{1} e^{-\bar{x}}}{a_{1}+\left(b_{1}+c_{1}\right) \bar{y}}\right) \\
& <\frac{\beta_{1} e^{-L_{1}}}{a_{1}+\left(b_{1}+c_{1}\right) L_{2}}+\left(1+\frac{\beta_{1} e^{-L_{1}}}{a_{1}+\left(b_{1}+c_{1}\right) L_{2}}\right) \\
& \times\left(\frac{c_{2}\left(\alpha_{2}+\left(\beta_{2}+\gamma_{2}\right) e^{-L_{2}}\right)}{\left(a_{2}+\left(b_{2}+c_{2}\right) L_{1}\right)^{2}}+\frac{\left(\beta_{2}+\gamma_{2}\right) e^{-L_{2}}}{a_{2}+\left(b_{2}+c_{2}\right) L_{1}}\right) \\
& +\frac{b_{2}\left(\alpha_{2}+\left(\beta_{2}+\gamma_{2}\right) e^{-L_{2}}\right)}{\left(a_{2}+\left(b_{2}+c_{2}\right) L_{1}\right)^{2}} \\
& \times\left(\frac{\left(b_{1}+c_{1}\right)\left(\alpha_{1}+\left(\beta_{1}+\gamma_{1}\right) e^{-L_{1}}\right)}{\left(a_{1}+\left(b_{1}+c_{1}\right) L_{2}\right)^{2}}\right. \\
& \left.+\frac{\gamma_{1} e^{-L_{1}}}{a_{1}+\left(b_{1}+c_{1}\right) L_{2}}\right)
\end{aligned}
$$

$<1$.

Then, by Rouche's Theorem, $\Phi(\lambda)$ and $\Phi(\lambda)-\Psi(\lambda)$ have the same number of zeroes in an open unit disk $|\lambda|<1$. Hence, all the roots of (31) satisfy $|\lambda|<1$, and it follows from Lemma 5 that the unique positive equilibrium point $(\bar{x}, \bar{y})$ of the system (5) is locally asymptotically stable.

Theorem 8. The unique positive equilibrium point $(\bar{x}, \bar{y})$ of system (5) is globally asymptotically stable, if the following condition is satisfied:

$$
\begin{aligned}
& \alpha_{1}+\left(\beta_{1}+\gamma_{1}\right) e^{-L_{1}}<\bar{x}\left(a_{1}+\left(b_{1}+c_{1}\right) L_{2}\right), \\
& \alpha_{2}+\left(\beta_{2}+\gamma_{2}\right) e^{-L_{2}}<\bar{y}\left(a_{2}+\left(b_{2}+c_{2}\right) L_{1}\right) .
\end{aligned}
$$


Proof. Arguing as in [18], we consider the following discrete time analogue of Lyapunov function:

$$
V_{n}=\bar{x}\left(\frac{x_{n}}{\bar{x}}-1-\ln \frac{x_{n}}{\bar{x}}\right)+\bar{y}\left(\frac{y_{n}}{\bar{y}}-1-\ln \frac{y_{n}}{\bar{y}}\right) .
$$

Then nonnegativity of $V_{n}$ follows from the following inequality:

$$
x-1-\ln x \geq 0 \quad \forall x>0 .
$$

Furthermore, we have

$$
\begin{aligned}
& -\ln \left(\frac{x_{n+1}}{x_{n}}\right)=\ln \left(1-\left(1-\frac{x_{n}}{x_{n+1}}\right)\right) \leq-\frac{x_{n+1}-x_{n}}{x_{n+1}}, \\
& -\ln \left(\frac{y_{n+1}}{y_{n}}\right)=\ln \left(1-\left(1-\frac{y_{n}}{y_{n+1}}\right)\right) \leq-\frac{y_{n+1}-y_{n}}{y_{n+1}} .
\end{aligned}
$$

Assume that (33) holds true; then, it follows that

$$
\begin{aligned}
& V_{n+1}-V_{n}=\bar{x}\left(\frac{x_{n+1}}{\bar{x}}-1-\ln \frac{x_{n+1}}{\bar{x}}\right) \\
& +\bar{y}\left(\frac{y_{n+1}}{\bar{y}}-1-\ln \frac{y_{n+1}}{\bar{y}}\right) \\
& -\bar{x}\left(\frac{x_{n}}{\bar{x}}-1-\ln \frac{x_{n}}{\bar{x}}\right)-\bar{y}\left(\frac{y_{n}}{\bar{y}}-1-\ln \frac{y_{n}}{\bar{y}}\right) \\
& \leq\left(x_{n+1}-x_{n}\right)+\left(y_{n+1}-y_{n}\right) \\
& -\frac{\bar{x}}{x_{n+1}}\left(x_{n+1}-x_{n}\right)-\frac{\bar{y}}{y_{n+1}}\left(y_{n+1}-y_{n}\right) \\
& =\left(x_{n+1}-x_{n}\right)\left(1-\frac{\bar{x}}{x_{n+1}}\right) \\
& +\left(y_{n+1}-y_{n}\right)\left(1-\frac{\bar{y}}{y_{n+1}}\right) \\
& \leq\left(U_{1}-L_{1}\right) \\
& \times\left(\frac{\alpha_{1}+\left(\beta_{1}+\gamma_{1}\right) e^{-L_{1}}-\bar{x}\left(a_{1}+\left(b_{1}+c_{1}\right) L_{2}\right)}{\alpha_{1}+\left(\beta_{1}+\gamma_{1}\right) e^{-L_{1}}}\right) \\
& +\left(U_{2}-L_{2}\right) \\
& \times\left(\frac{\alpha_{2}+\left(\beta_{2}+\gamma_{2}\right) e^{-L_{2}}-\bar{y}\left(a_{2}+\left(b_{2}+c_{2}\right) L_{1}\right)}{\alpha_{2}+\left(\beta_{2}+\gamma_{2}\right) e^{-L_{2}}}\right) \\
& \leq 0
\end{aligned}
$$

for all $n \geq 0$ so that $V_{n} \geq 0$ is monotonically decreasing sequence. It follows that $\lim _{n \rightarrow \infty} V_{n} \geq 0$. Hence, we obtain that

$$
\lim _{n \rightarrow \infty}\left(V_{n+1}-V_{n}\right)=0 .
$$

Then it follows that $\lim _{n \rightarrow \infty} x_{n+1}=\bar{x}$ and $\lim _{n \rightarrow \infty} y_{n+1}=\bar{y}$. Furthermore, $V_{n} \leq V_{0}$ for all $n \geq 0$, which gives that $(\bar{x}, \bar{y}) \in$ $\left[L_{1}, U_{1}\right] \times\left[L_{2}, U_{2}\right]$ is uniformly stable. Hence, unique positive equilibrium point $(\bar{x}, \bar{y}) \in\left[L_{1}, U_{1}\right] \times\left[L_{2}, U_{2}\right]$ of system (5) is globally asymptotically stable.
2.2. Rate of Convergence. In this section we will determine the rate of convergence of a solution that converges to the unique positive equilibrium point of the system (5).

The following result gives the rate of convergence of solutions of a system of difference equations:

$$
X_{n+1}=(A+B(n)) X_{n} \text {, }
$$

where $X_{n}$ is an $m$-dimensional vector, $A \in C^{m \times m}$ is a constant matrix, and $B: \mathbb{Z}^{+} \rightarrow C^{m \times m}$ is a matrix function satisfying

$$
\|B(n)\| \longrightarrow 0
$$

as $n \rightarrow \infty$, where $\|\cdot\|$ denotes any matrix norm which is associated with the vector norm

$$
\|(x, y)\|=\sqrt{x^{2}+y^{2}} .
$$

Proposition 9 ((Perron's Theorem) [19]). Suppose that condition (40) holds. If $X_{n}$ is a solution of (39), then either $X_{n}=0$ for all large $n$ or

$$
\rho=\lim _{n \rightarrow \infty}\left(\left\|X_{n}\right\|\right)^{1 / n}
$$

exists and is equal to the modulus of one of the eigenvalues of matrix A.

Proposition 10 (see [19]). Suppose that condition (40) holds. If $X_{n}$ is a solution of (39), then either $X_{n}=0$ for all large $n$ or

$$
\rho=\lim _{n \rightarrow \infty} \frac{\left\|X_{n+1}\right\|}{\left\|X_{n}\right\|}
$$

exists and is equal to the modulus of one of the eigenvalues of matrix A.

Let $\left\{\left(x_{n}, y_{n}\right)\right\}$ be any solution of the system (5) such that $\lim _{n \rightarrow \infty} x_{n}=\bar{x}$ and $\lim _{n \rightarrow \infty} y_{n}=\bar{y}$, where $\bar{x} \in\left[L_{1}, U_{1}\right]$ and $\bar{y} \in\left[L_{2}, U_{2}\right]$. To find the error terms, one has from the system (5)

$$
\begin{aligned}
x_{n+1} & -\bar{x} \\
= & \frac{\alpha_{1}+\beta_{1} e^{-x_{n}}+\gamma_{1} e^{-x_{n-1}}}{a_{1}+b_{1} y_{n}+c_{1} y_{n-1}}-\frac{\alpha_{1}+\left(\beta_{1}+\gamma_{1}\right) e^{-\bar{x}}}{a_{1}+\left(b_{1}+c_{1}\right) \bar{y}} \\
= & \frac{\beta_{1}\left(e^{-x_{n}}-e^{-\bar{x}}\right)}{\left(a_{1}+b_{1} y_{n}+c_{1} y_{n-1}\right)\left(x_{n}-\bar{x}\right)}\left(x_{n}-\bar{x}\right) \\
& -\frac{b_{1}\left(\alpha_{1}+\left(\beta_{1}+\gamma_{1}\right) e^{-\bar{x}}\right)}{\left(a_{1}+\left(b_{1}+c_{1}\right) \bar{y}\right)\left(a_{1}+b_{1} y_{n}+c_{1} y_{n-1}\right)}\left(y_{n}-\bar{y}\right) \\
& +\frac{\gamma_{1}\left(e^{-x_{n-1}}-e^{-\bar{x}}\right)}{\left(a_{1}+b_{1} y_{n}+c_{1} y_{n-1}\right)\left(x_{n-1}-\bar{x}\right)}\left(x_{n-1}-\bar{x}\right) \\
& -\frac{c_{1}\left(\alpha_{1}+\left(\beta_{1}+\gamma_{1}\right) e^{-\bar{x}}\right)}{\left(a_{1}+\left(b_{1}+c_{1}\right) \bar{y}\right)\left(a_{1}+b_{1} y_{n}+c_{1} y_{n-1}\right)}\left(y_{n-1}-\bar{y}\right),
\end{aligned}
$$




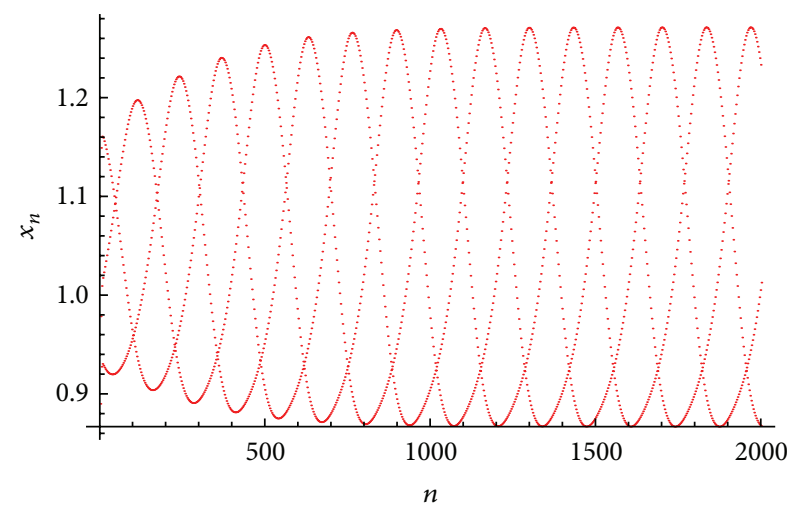

(a) Plot of $x_{n}$ for the system (50)

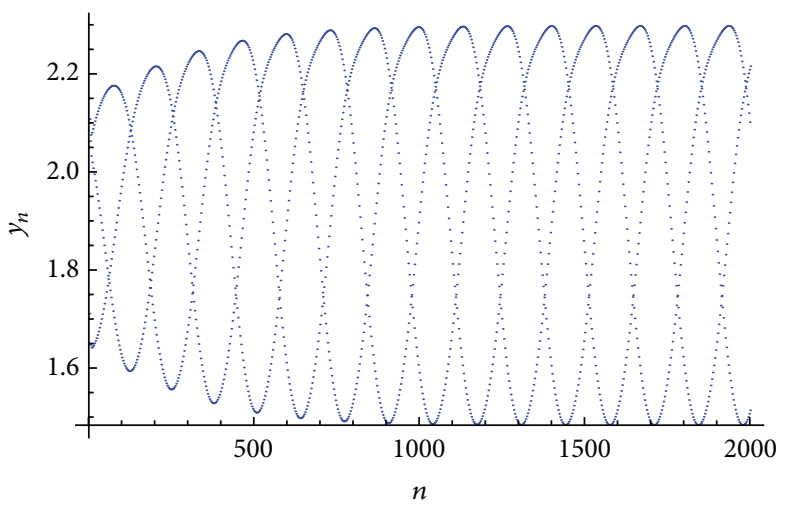

(b) Plot of $y_{n}$ for the system (50)

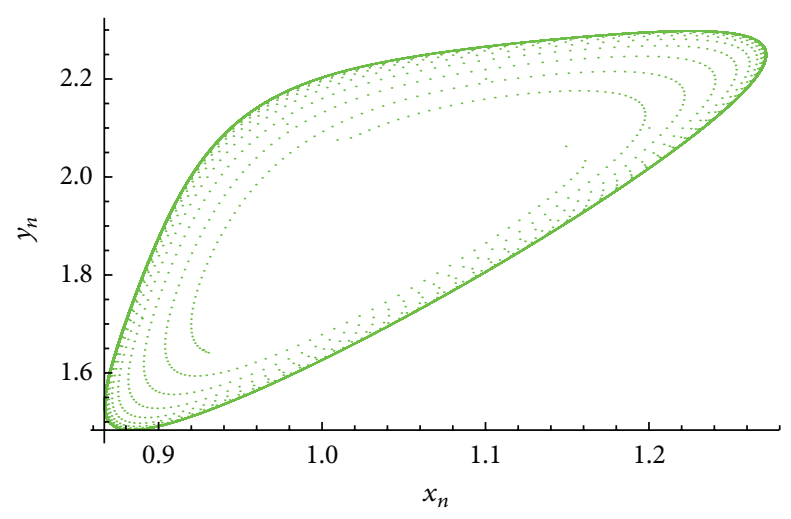

(c) Phase portrait of system (50)

Figure 1: Plots for the system (50).

$y_{n+1}-\bar{y}$

$$
\begin{aligned}
= & \frac{\alpha_{2}+\beta_{2} e^{-y_{n}}+\gamma_{2} e^{-y_{n-1}}}{a_{2}+b_{2} x_{n}+c_{2} x_{n-1}}-\frac{\alpha_{2}+\left(\beta_{2}+\gamma_{2}\right) e^{-\bar{y}}}{a_{2}+\left(b_{2}+c_{2}\right) \bar{x}} \\
= & \frac{\beta_{2}\left(e^{-y_{n}}-e^{-\bar{y}}\right)}{\left(a_{2}+b_{2} x_{n}+c_{2} x_{n-1}\right)\left(y_{n}-\bar{x}\right)}\left(y_{n}-\bar{y}\right) \\
& -\frac{b_{2}\left(\alpha_{2}+\left(\beta_{2}+\gamma_{2}\right) e^{-\bar{y}}\right)}{\left(a_{2}+\left(b_{2}+c_{2}\right) \bar{x}\right)\left(a_{2}+b_{2} x_{n}+c_{2} x_{n-1}\right)}\left(x_{n}-\bar{x}\right) \\
& +\frac{\gamma_{2}\left(e^{-y_{n-1}}-e^{-\bar{y}}\right)}{\left(a_{2}+b_{2} x_{n}+c_{2} x_{n-1}\right)\left(y_{n-1}-\bar{y}\right)}\left(y_{n-1}-\bar{y}\right) \\
& -\frac{c_{2}\left(\alpha_{2}+\left(\beta_{2}+\gamma_{2}\right) e^{-\bar{y}}\right)}{\left(a_{2}+\left(b_{2}+c_{2}\right) \bar{x}\right)\left(a_{2}+b_{2} x_{n}+c_{2} x_{n-1}\right)}\left(x_{n-1}-\bar{x}\right) .
\end{aligned}
$$

Let $e_{n}^{1}=x_{n}-\bar{x}$ and $e_{n}^{2}=y_{n}-\bar{y}$; then, one has

$$
\begin{aligned}
& e_{n+1}^{1}=a_{n} e_{n}^{1}+b_{n} e_{n}^{2}+c_{n} e_{n-1}^{1}+d_{n} e_{n-1}^{2}, \\
& e_{n+1}^{2}=f_{n} e_{n}^{1}+g_{n} e_{n}^{2}+h_{n} e_{n-1}^{1}+k_{n} e_{n-1}^{2},
\end{aligned}
$$

where

$$
\begin{aligned}
& a_{n}=\frac{\beta_{1}\left(e^{-x_{n}}-e^{-\bar{x}}\right)}{\left(a_{1}+b_{1} y_{n}+c_{1} y_{n-1}\right)\left(x_{n}-\bar{x}\right)}, \\
& b_{n}=-\frac{b_{1}\left(\alpha_{1}+\left(\beta_{1}+\gamma_{1}\right) e^{-\bar{x}}\right)}{\left(a_{1}+\left(b_{1}+c_{1}\right) \bar{y}\right)\left(a_{1}+b_{1} y_{n}+c_{1} y_{n-1}\right)} \text {, } \\
& c_{n}=\frac{\gamma_{1}\left(e^{-x_{n-1}}-e^{-\bar{x}}\right)}{\left(a_{1}+b_{1} y_{n}+c_{1} y_{n-1}\right)\left(x_{n-1}-\bar{x}\right)} \text {, } \\
& d_{n}=-\frac{c_{1}\left(\alpha_{1}+\left(\beta_{1}+\gamma_{1}\right) e^{-\bar{x}}\right)}{\left(a_{1}+\left(b_{1}+c_{1}\right) \bar{y}\right)\left(a_{1}+b_{1} y_{n}+c_{1} y_{n-1}\right)} \text {, } \\
& f_{n}=-\frac{b_{2}\left(\alpha_{2}+\left(\beta_{2}+\gamma_{2}\right) e^{-\bar{y}}\right)}{\left(a_{2}+\left(b_{2}+c_{2}\right) \bar{x}\right)\left(a_{2}+b_{2} x_{n}+c_{2} x_{n-1}\right)} \text {, } \\
& g_{n}=\frac{\beta_{2}\left(e^{-y_{n}}-e^{-\bar{y}}\right)}{\left(a_{2}+b_{2} x_{n}+c_{2} x_{n-1}\right)\left(y_{n}-\bar{x}\right)}, \\
& h_{n}=-\frac{c_{2}\left(\alpha_{2}+\left(\beta_{2}+\gamma_{2}\right) e^{-\bar{y}}\right)}{\left(a_{2}+\left(b_{2}+c_{2}\right) \bar{x}\right)\left(a_{2}+b_{2} x_{n}+c_{2} x_{n-1}\right)} \text {, } \\
& k_{n}=\frac{\gamma_{2}\left(e^{-y_{n-1}}-e^{-\bar{y}}\right)}{\left(a_{2}+b_{2} x_{n}+c_{2} x_{n-1}\right)\left(y_{n-1}-\bar{y}\right)} \text {. }
\end{aligned}
$$




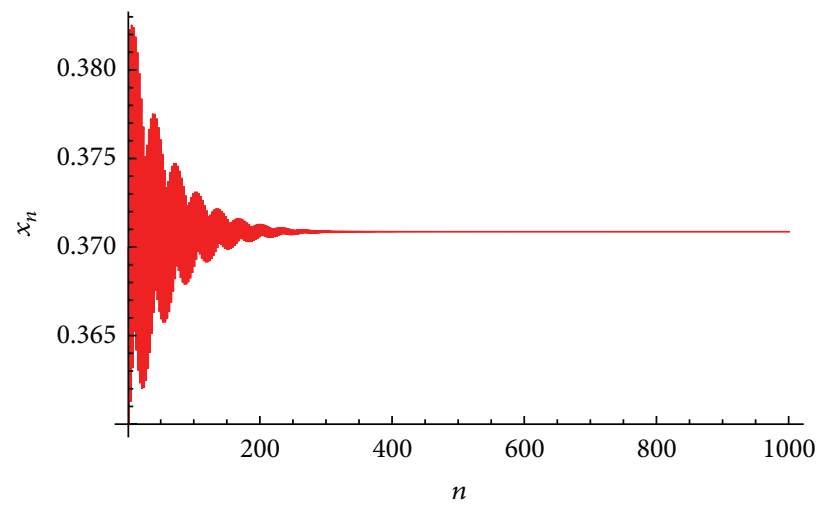

(a) Plot of $x_{n}$ for the system (51)

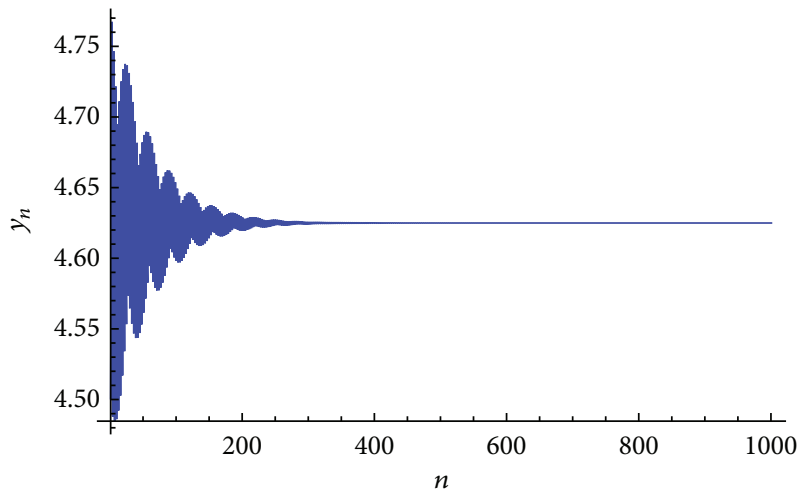

(b) Plot of $y_{n}$ for the system (51)

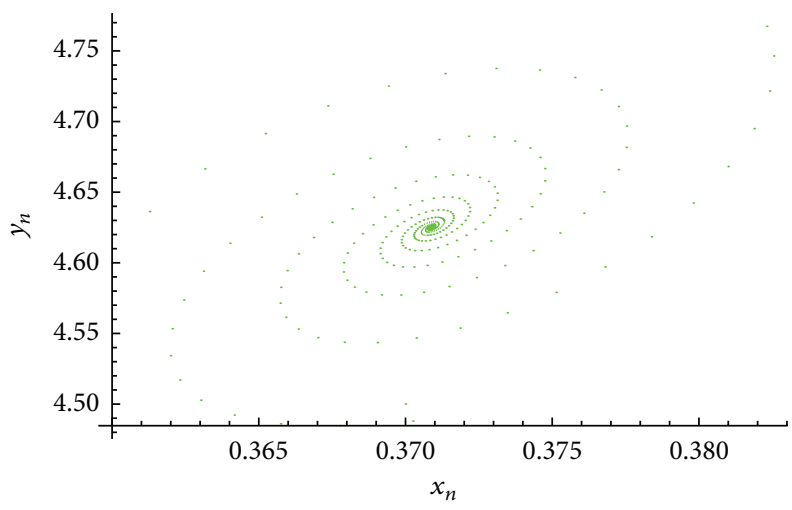

(c) An attractor of the system (51)

Figure 2: Plots for the system (51).

Moreover,

$$
\begin{aligned}
& \lim _{n \rightarrow \infty} a_{n}=-\frac{\beta_{1} e^{-\bar{x}}}{a_{1}+\left(b_{1}+c_{1}\right) \bar{y}}=A_{1}, \\
& \lim _{n \rightarrow \infty} b_{n}=-\frac{b_{1}\left(\alpha_{1}+\left(\beta_{1}+\gamma_{1}\right) e^{-\bar{x}}\right)}{\left(a_{1}+\left(b_{1}+c_{1}\right) \bar{y}\right)^{2}}=A_{2}, \\
& \lim _{n \rightarrow \infty} c_{n}=-\frac{\gamma_{1} e^{-\bar{x}}}{a_{1}+\left(b_{1}+c_{1}\right) \bar{y}}=A_{3}, \\
& \lim _{n \rightarrow \infty} d_{n}=-\frac{c_{1}\left(\alpha_{1}+\left(\beta_{1}+\gamma_{1}\right) e^{-\bar{x}}\right)}{\left(a_{1}+\left(b_{1}+c_{1}\right) \bar{y}\right)^{2}}=A_{4}, \\
& \lim _{n \rightarrow \infty} f_{n}=-\frac{b_{2}\left(\alpha_{2}+\left(\beta_{2}+\gamma_{2}\right) e^{-\bar{y}}\right)}{\left(a_{2}+\left(b_{2}+c_{2}\right) \bar{x}\right)^{2}}=B_{1}, \\
& \lim _{n \rightarrow \infty} g_{n}=-\frac{\beta_{2} e^{-\bar{y}}}{a_{2}+\left(b_{2}+c_{2}\right) \bar{x}}=B_{2}, \\
& \lim _{n \rightarrow \infty} h_{n}=-\frac{c_{2}\left(\alpha_{2}+\left(\beta_{2}+\gamma_{2}\right) e^{-\bar{y}}\right)}{\left(a_{2}+\left(b_{2}+c_{2}\right) \bar{x}\right)^{2}}=B_{3}, \\
& \lim _{n \rightarrow \infty} k_{n}=-\frac{\gamma_{2} e^{-\bar{y}}}{a_{2}+\left(b_{2}+c_{2}\right) \bar{x}}=B_{4} .
\end{aligned}
$$

Now the limiting system of error terms can be written as

$$
\left[\begin{array}{c}
e_{n+1}^{1} \\
e_{n+1}^{2} \\
e_{n}^{1} \\
e_{n}^{2}
\end{array}\right]=\left[\begin{array}{cccc}
A_{1} & A_{2} & A_{3} & A_{4} \\
B_{1} & B_{2} & B_{3} & B_{4} \\
1 & 0 & 0 & 0 \\
0 & 1 & 0 & 0
\end{array}\right]\left[\begin{array}{c}
e_{n}^{1} \\
e_{n}^{2} \\
e_{n-1}^{1} \\
e_{n-1}^{2}
\end{array}\right]
$$

which is similar to linearized system of (5) about the equilibrium point $(\bar{x}, \bar{y})$.

Using Proposition 9, one has the following result.

Theorem 11. Assume that $\left\{\left(x_{n}, y_{n}\right)\right\}$ is a positive solution of the system (5) such that $\lim _{n \rightarrow \infty} x_{n}=\bar{x}$, and $\lim _{n \rightarrow \infty} y_{n}=\bar{y}$, where $\bar{x} \in\left[L_{1}, U_{1}\right]$ and $\bar{y} \in\left[L_{2}, U_{2}\right]$. Then, the error vector $e_{n}=\left(\begin{array}{c}e_{n}^{1} \\ e_{n}^{2} \\ e_{n-1}^{1} \\ e_{n-1}^{2}\end{array}\right)$ of every solution of (5) satisfies both of the following asymptotic relations:

$$
\begin{gathered}
\lim _{n \rightarrow \infty}\left(\left\|e_{n}\right\|\right)^{1 / n}=\left|\lambda_{1,2,3,4} F_{J}(\bar{x}, \bar{y})\right|, \\
\lim _{n \rightarrow \infty} \frac{\left\|e_{n+1}\right\|}{\left\|e_{n}\right\|}=\left|\lambda_{1,2,3,4} F_{J}(\bar{x}, \bar{y})\right|,
\end{gathered}
$$

where $\lambda_{1,2,3,4} F_{J}(\bar{x}, \bar{y})$ are the characteristic roots of Jacobian matrix $F_{J}(\bar{x}, \bar{y})$. 


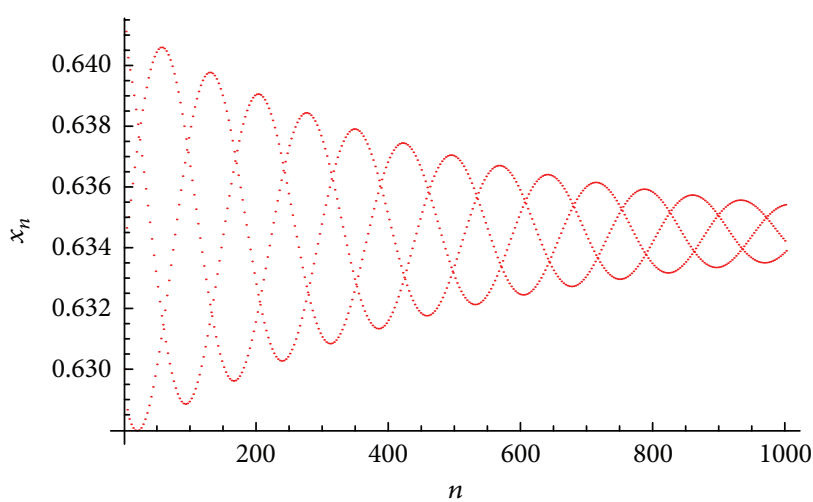

(a) Plot of $x_{n}$ for the system (52)

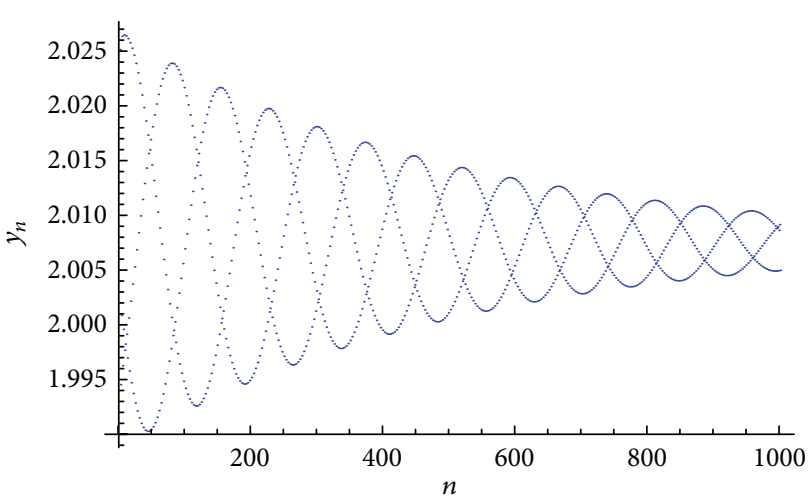

(b) Plot of $y_{n}$ for the system (52)

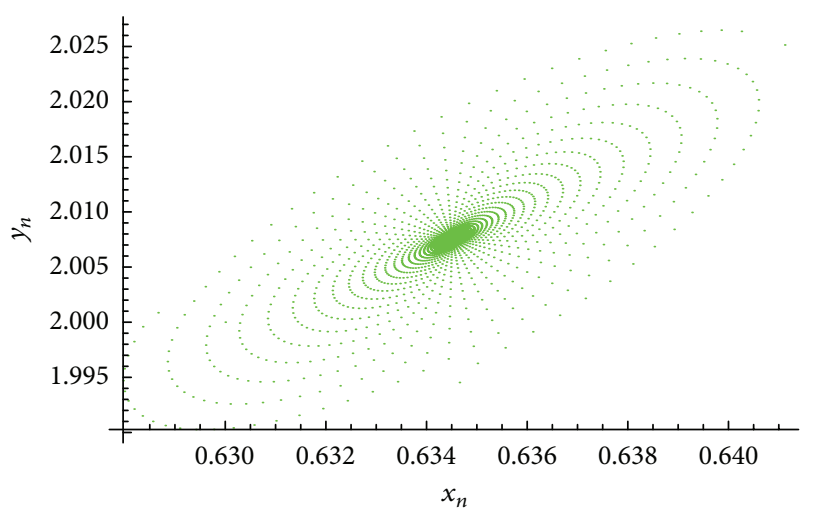

(c) An attractor of the system (52)

FIGURE 3: Plots for the system (52).

2.3. Examples. In order to verify our theoretical results and to support our theoretical discussions, we consider several interesting numerical examples in this section. These examples represent different types of qualitative behavior of solutions to the system of nonlinear difference equations (5). The first and last examples show that positive equilibrium of system (5) is unstable with suitable parametric choices. Moreover, from the remaining examples it is clear that unique positive equilibrium point of system (5) is globally asymptotically stable with different parametric values.

Example 1. Let $\alpha_{1}=6.5, \beta_{1}=8.5, \gamma_{1}=12.3, a_{1}=1.6, b_{1}=$ $5.02, c_{1}=1.2, \alpha_{2}=3.5, \beta_{2}=5.5, \gamma_{2}=1.3, a_{2}=0.1, b_{2}=$ 0.003 , and $c_{2}=2.2$. Then, system (5) can be written as

$$
\begin{gathered}
x_{n+1}=\frac{6.5+12.3 e^{-x_{n}}+8.5 e^{-x_{n-1}}}{1.6+1.2 y_{n}+5.02 y_{n-1}}, \\
y_{n+1}=\frac{3.5+1.3 e^{-y_{n}}+5.5 e^{-y_{n-1}}}{0.1+2.2 x_{n}+0.003 x_{n-1}},
\end{gathered}
$$

with initial conditions $x_{-1}=1.2, x_{0}=1.1, y_{-1}=2.1$, and $y_{0}=2.2$.

In this case the positive equilibrium point of the system (50) is unstable. Moreover, in Figure 1 the plot of $x_{n}$ is shown in Figure 1(a), the plot of $y_{n}$ is shown in Figure 1(b), and a phase portrait of the system (50) is shown in Figure 1(c).
Example 2. Let $\alpha_{1}=1.5, \beta_{1}=7.5, \gamma_{1}=13.5, a_{1}=2.1, b_{1}=$ $7.92, c_{1}=0.95, \alpha_{2}=4.1, \beta_{2}=7.2, \gamma_{2}=16.7, a_{2}=0.01$, $b_{2}=0.2$, and $c_{2}=2.3$. Then, system (5) can be written as

$$
\begin{aligned}
& x_{n+1}=\frac{1.5+7.5 e^{-x_{n}}+13.5 e^{-x_{n-1}}}{2.1+7.92 y_{n}+0.95 y_{n-1}}, \\
& y_{n+1}=\frac{4.1+7.2 e^{-y_{n}}+16.7 e^{-y_{n-1}}}{0.01+0.2 x_{n}+2.3 x_{n-1}},
\end{aligned}
$$

with initial conditions $x_{-1}=0.37, x_{0}=0.36, y_{-1}=4.5$, and $y_{0}=4.6$.

In this case the unique positive equilibrium point of the system (51) is given by $(\bar{x}, \bar{y})=(0.370864,4.62495)$. Moreover, in Figure 2 the plot of $x_{n}$ is shown in Figure 2(a), the plot of $y_{n}$ is shown in Figure 2(b), and an attractor of the system (51) is shown in Figure 2(c).

Example 3. Let $\alpha_{1}=8.98, \beta_{1}=75, \gamma_{1}=135, a_{1}=21, b_{1}=79$, $c_{1}=5, \alpha_{2}=41, \beta_{2}=71.9, \gamma_{2}=16, a_{2}=6, b_{2}=2$, and $c_{2}=30$. Then, system (5) can be written as

$$
\begin{gathered}
x_{n+1}=\frac{8.98+75 e^{-x_{n}}+135 e^{-x_{n-1}}}{21+79 y_{n}+5 y_{n-1}}, \\
y_{n+1}=\frac{41+71.9 e^{-y_{n}}+16 e^{-y_{n-1}}}{6+2 x_{n}+30 x_{n-1}},
\end{gathered}
$$




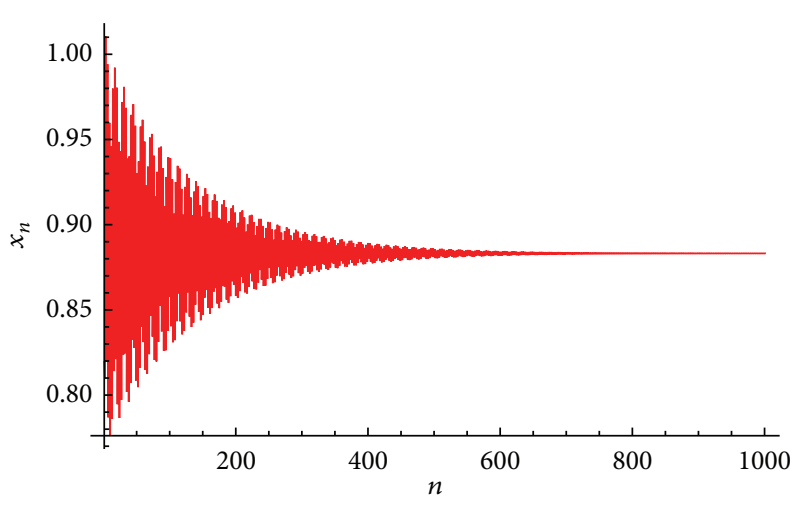

(a) Plot of $x_{n}$ for the system (53)

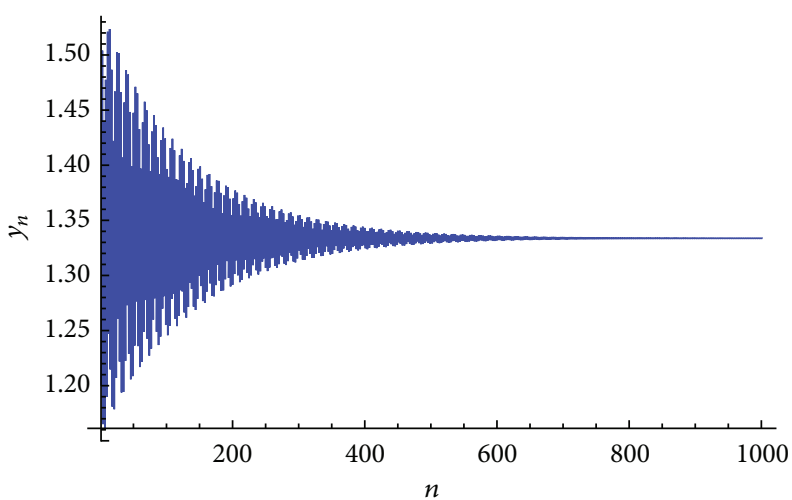

(b) Plot of $y_{n}$ for the system (53)

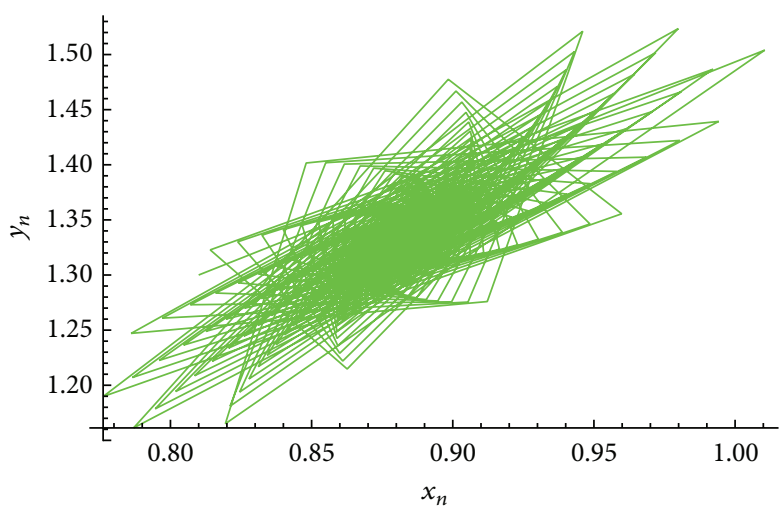

(c) An attractor of the system (53)

FIgURE 4: Plots for the system (53).

with initial conditions $x_{-1}=0.634, x_{0}=0.63, y_{-1}=1.99$, and $y_{0}=2$.

In this case the unique positive equilibrium point of the system $(52)$ is given by $(\bar{x}, \bar{y})=(0.634497,2.00755)$. Moreover, in Figure 3 the plot of $x_{n}$ is shown in Figure 3(a), the plot of $y_{n}$ is shown in Figure 3(b), and an attractor of the system (52) is shown in Figure 3(c).

Example 4. Let $\alpha_{1}=23.8, \beta_{1}=350, \gamma_{1}=66, a_{1}=55, b_{1}=14$, $c_{1}=111, \alpha_{2}=66, \beta_{2}=35, \gamma_{2}=87, a_{2}=1.7, b_{2}=80$, and $c_{2}=1.4$. Then, system (5) can be written as

$$
\begin{gathered}
x_{n+1}=\frac{23.8+350 e^{-x_{n}}+66 e^{-x_{n-1}}}{55+14 y_{n}+111 y_{n-1}}, \\
y_{n+1}=\frac{66+35 e^{-y_{n}}+87 e^{-y_{n-1}}}{1.7+80 x_{n}+1.4 x_{n-1}},
\end{gathered}
$$

with initial conditions $x_{-1}=0.82, x_{0}=0.81, y_{-1}=1.2$, and $y_{0}=1.3$.

In this case the unique positive equilibrium point of the system $(53)$ is given by $(\bar{x}, \bar{y})=(0.883148,1.33372)$. Moreover, in Figure 4 the plot of $x_{n}$ is shown in Figure 4(a), the plot of $y_{n}$ is shown in Figure $4(\mathrm{~b})$, and an attractor of system (53) is shown in Figure 4(c).
Example 5. Let $\alpha_{1}=8, \beta_{1}=3, \gamma_{1}=6, a_{1}=0.05, b_{1}=4$, $c_{1}=16, \alpha_{2}=9, \beta_{2}=17, \gamma_{2}=7, a_{2}=0.07, b_{2}=22$, and $c_{2}=0.2$. Then, system (5) can be written as

$$
\begin{gathered}
x_{n+1}=\frac{8+3 e^{-x_{n}}+6 e^{-x_{n-1}}}{0.05+4 y_{n}+16 y_{n-1}}, \\
y_{n+1}=\frac{9+17 e^{-y_{n}}+7 e^{-y_{n-1}}}{0.07+22 x_{n}+0.2 x_{n-1}},
\end{gathered}
$$

with initial conditions $x_{-1}=0.5, x_{0}=0.4, y_{-1}=1.2$, and $y_{0}=1.3$.

In this case the positive equilibrium point of the system (54) is unstable. Moreover, in Figure 5 the plot of $x_{n}$ is shown in Figure 5(a), the plot of $y_{n}$ is shown in Figure 5(b), and a phase portrait of system (54) is shown in Figure 5(c).

\section{Concluding Remarks}

In literature several articles are related to qualitative behavior of exponential systems of rational difference equations. It is a very interesting mathematical problem to study the dynamics of such systems because these are closely related to models in population dynamics and biological sciences. This work is related to qualitative behavior of an exponential system of second-order rational difference equations. We have investigated the existence and uniqueness of positive steady 


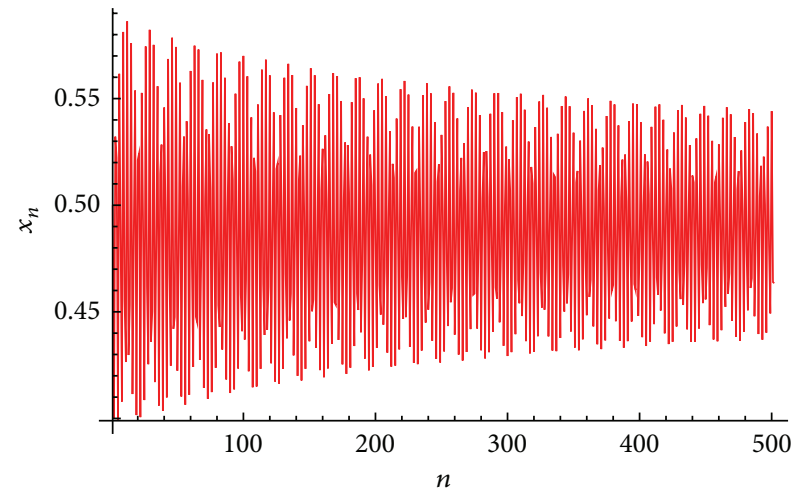

(a) Plot of $x_{n}$ for the system (54)

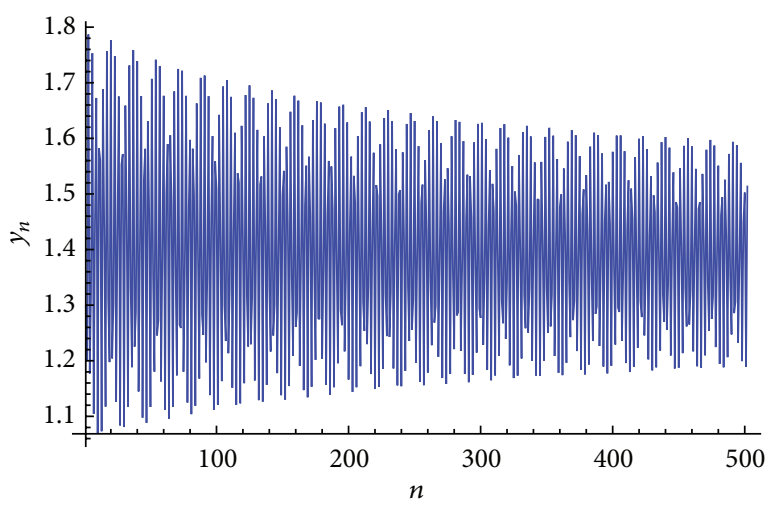

(b) Plot of $y_{n}$ for the system (54)

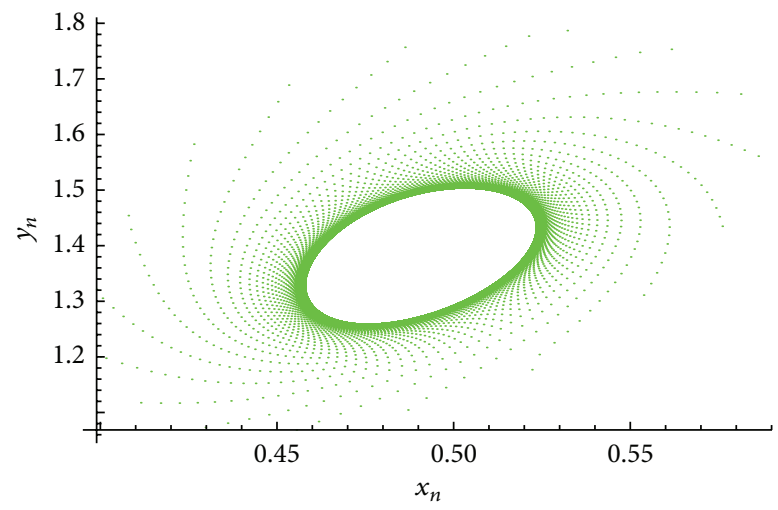

(c) Phase portrait of system (54)

FIGURE 5: Plots for the system (54).

state of system (5). Under certain parametric conditions the boundedness and persistence of positive solutions are proved. Moreover, we have shown that unique positive equilibrium point of system (5) is locally as well as globally asymptotically stable. The main objective of dynamical systems theory is to predict the global behavior of a system based on the knowledge of its present state. An approach to this problem consists of determining the possible global behaviors of the system and determining which parametric conditions lead to these long-term behaviors. By constructing a discrete Lyapunov function, we have obtained the global asymptotic stability of the positive equilibrium of (5). Furthermore, rate of convergence of positive solutions of (5) which converge to its unique positive equilibrium point is demonstrated. Finally, some illustrative examples are provided to support our theoretical discussion.

\section{Conflict of Interests}

The authors declare that they have no conflict of interests regarding the publication of this paper.

\section{Acknowledgments}

The authors thank the main editor and the anonymous referees for their valuable comments and suggestions leading to improvement of this paper. This work was supported by the Higher Education Commission of Pakistan.

\section{References}

[1] E. El-Metwally, E. A. Grove, G. Ladas, R. Levins, and M. Radin, "On the difference equation $x_{n+1}=a+\beta x_{n-1} e^{-x_{n}}$ ", Nonlinear Analysis, vol. 47, pp. 4623-4634, 2001.

[2] G. Papaschinopoulos, M. A. Radin, and C. J. Schinas, "On the system of two difference equations of exponential form: $x_{n+1}=$ $a+b x_{n-1} e^{-y n}, y_{n+1}=c+d y_{n-1} e^{-x n}$, Mathematical and Computer Modelling, vol. 54, no. 11-12, pp. 2969-2977, 2011.

[3] G. Papaschinopoulos, M. A. Radin, and C. J. Schinas, "Study of the asymptotic behavior of the solutions of three systems of difference equations of exponential form," Applied Mathematics and Computation, vol. 218, no. 9, pp. 5310-5318, 2012.

[4] G. Papaschinopoulos and C. J. Schinas, "On the dynamics of two exponential type systems of difference equations," Computers \& Mathematics with Applications, vol. 64, no. 7, pp. 2326-2334, 2012.

[5] Q. Din, "Global stability of a population model," Chaos, Solitons \& Fractals, vol. 59, pp. 119-128, 2014.

[6] Q. Din and E. M. Elsayed, "Stability analysis of a discrete ecological model," Computational Ecology and Software, vol. 4, no. 2, pp. 89-103, 2014.

[7] M. R. S. Kulenovic and G. Ladas, Dynamics of Second Order Rational Difference Equations, Chapman \& Hall, CRC, 2002. 
[8] E. A. Grove and G. Ladas, Periodicities in Nonlinear Difference Equations, Chapman and Hall/CRC Press, Boca Raton, Fla, USA, 2004.

[9] H. Sedaghat, Nonlinear Difference Equations: Theory with Applications to Social Science Models, Kluwer Academic, Dordrecht, The Netherlands, 2003.

[10] S. Ahmad, "On the nonautonomous Volterra-Lotka competition equations," Proceedings of the American Mathematical Society, vol. 117, no. 1, pp. 199-204, 1993.

[11] X. Tang and X. Zou, "On positive periodic solutions of LotkaVolterra competition systems with deviating arguments," Proceedings of the American Mathematical Society, vol. 134, no. 10, pp. 2967-2974, 2006.

[12] Z. Zhou and X. Zou, "Stable periodic solutions in a discrete periodic logistic equation," Applied Mathematics Letters, vol. 16, no. 2, pp. 165-171, 2003.

[13] X. Liu, "A note on the existence of periodic solutions in discrete predator-prey models," Applied Mathematical Modelling: Simulation and Computation for Engineering and Environmental Systems, vol. 34, no. 9, pp. 2477-2483, 2010.

[14] Q. Din, "Dynamics of a discrete Lotka-Volterra model," Advances in Difference Equations, vol. 2013, article 95, 2013.

[15] Q. Din and T. Donchev, "Global character of a host-parasite model," Chaos, Solitons \& Fractals, vol. 54, pp. 1-7, 2013.

[16] Q. Din, "Stability analysis of a biological network," Network Biology, vol. 4, no. 3, pp. 123-129, 2014.

[17] Q. Din, T. F. Ibrahim, and K. A. Khan, "Behavior of a competitive system of second-order difference equations," The Scientific World Journal, vol. 2014, Article ID 283982, 9 pages, 2014.

[18] Y. Enatsu, Y. Nakata, and Y. Muroya, "Global stability for a class of discrete SIR epidemic models," Mathematical Biosciences and Engineering, vol. 7, no. 2, pp. 347-361, 2010.

[19] M. Pituk, "More on Poincaré's and Perron's theorems for difference equations," Journal of Difference Equations and Applications, vol. 8, no. 3, pp. 201-216, 2002. 


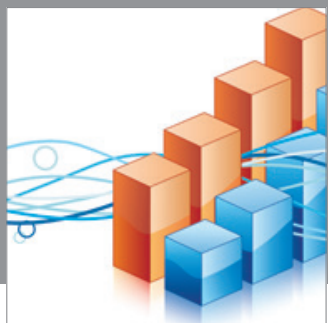

Advances in

Operations Research

mansans

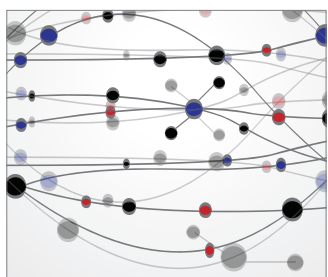

The Scientific World Journal
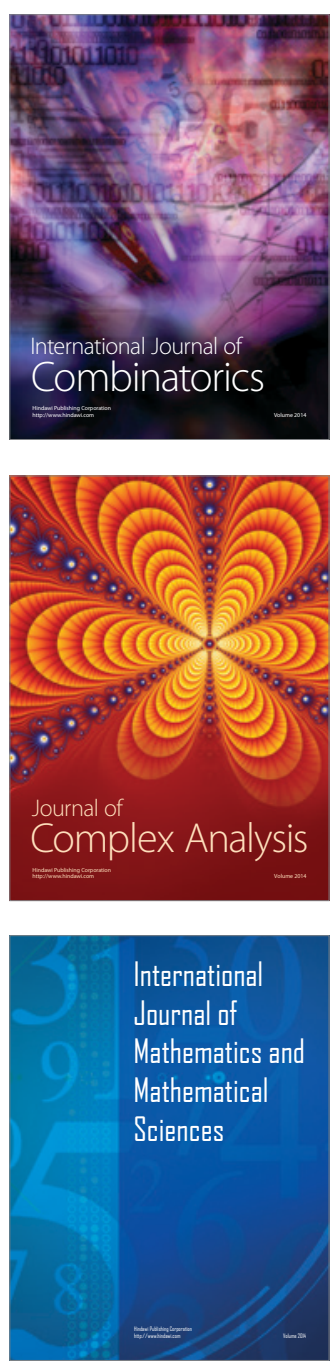
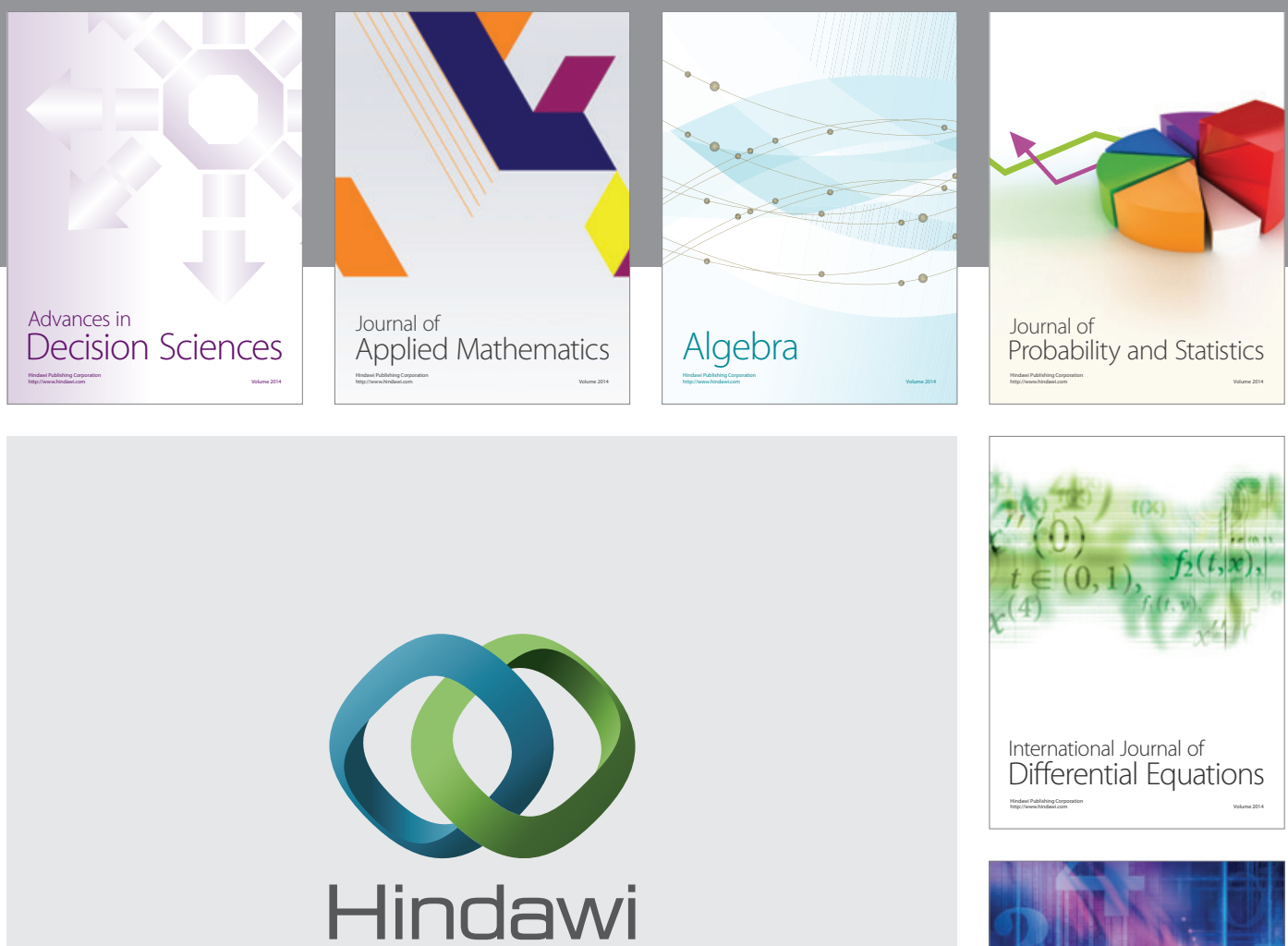

Submit your manuscripts at http://www.hindawi.com
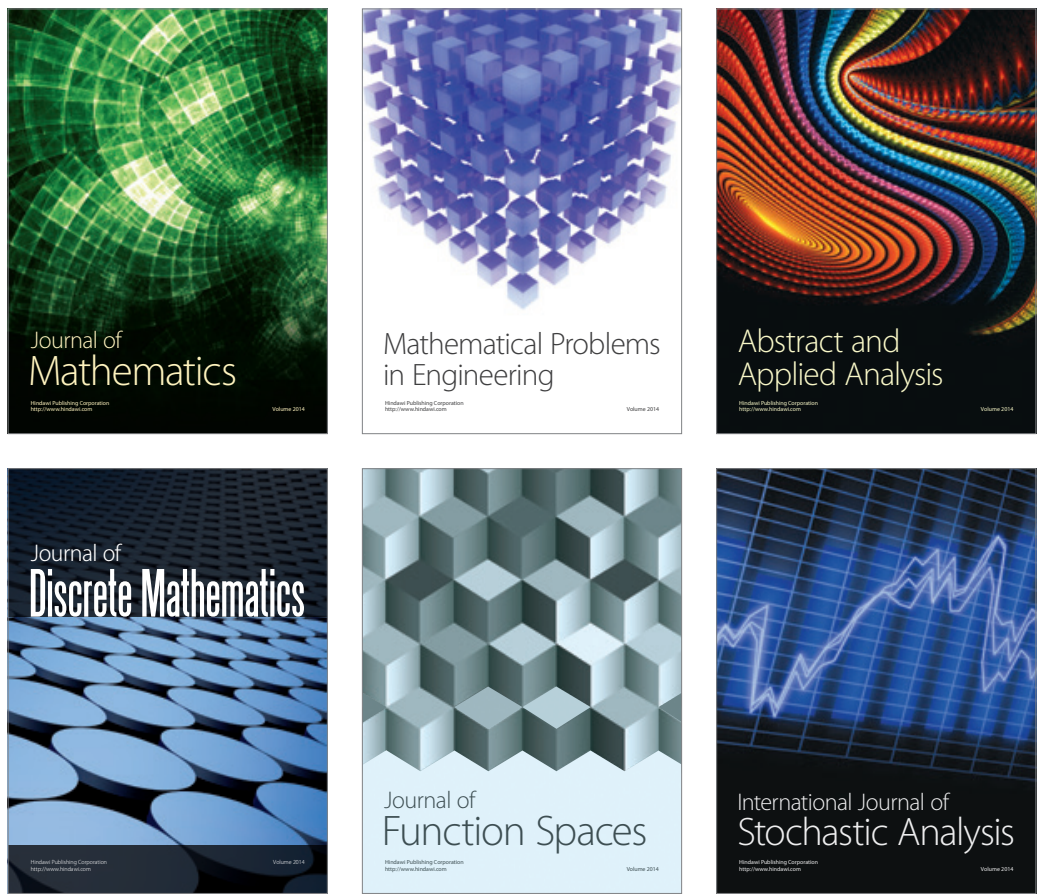

Journal of

Function Spaces

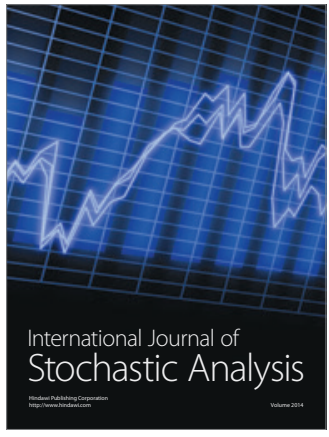

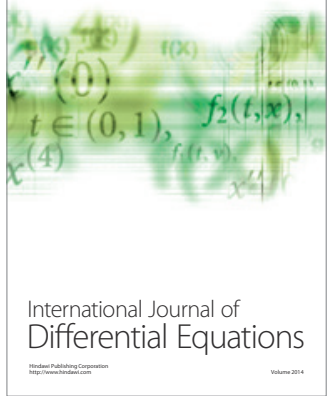
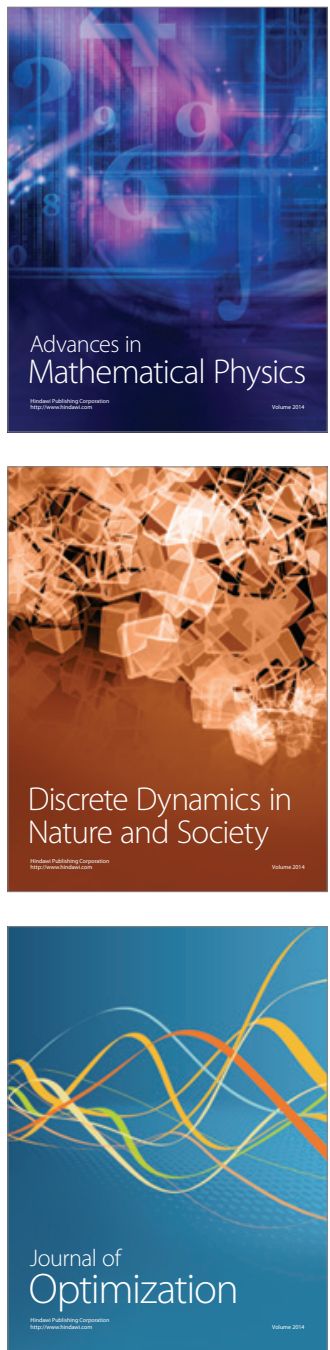